\title{
Influence of the Lossy Compression JPEG2000 standard on the Deformation of PSF
}

\author{
P. Páta
}

\begin{abstract}
This paper deals with the influence of lossy compression algorithms on the deformation of the point spread function (PSF) of imaging systems in astronomy. Lossy compression algorithms reduce irrelevant information in image functions, and their application distorts the image function. Astronomical images have typical specific properties - high grayscale bit depth, size, noise occurrence and special processing algorithms. They belong to the class of scientific images as well as medical or similar. Their processing and compression is quite different from the classical approach of multimedia image processing. The influence of the JPEG2000 coder on the deformation of PSF is presented in this paper.
\end{abstract}

Keywords: Astronomical lossy image compression, PSF deformation, Astronomical Context Coder (ACC), JPEG 2000.

\section{Introduction}

This paper deals with influence of the lossy compression standard JPEG 2000 on the deformation of the point spread function (PSF) of the imaging system. JPEG200 is a lossy compression standard exploiting the wavelet approach [4]. Image compression based on the wavelet transform is nowadays very popular because of its nice properties. The whole imaging system can be described using a model based on the point spread function. This approach demonstrates the influence of each part of the system on the quality of the acquired image. In the case of a system with linear and space invariant parameters the final PSF can be expressed as a convolution of the PSF of the parts of the system

$$
\begin{aligned}
& P S F(x, y)=P S F_{\text {air }}(x, y) \cdot P S F_{\text {optics }}(x, y) \cdot \\
& P S F_{\text {sensor }}(x, y) \cdot P S F_{I P}(x, y) \cdot P S F_{\text {compr }}(x, y),
\end{aligned}
$$

where $P S F_{\text {air }}(x, y)$ is the point spread function of the Earth's atmosphere, $P S F_{\text {optics }}(x, y)$ is the influence of the system optics, $P S F_{\text {sensor }}(x, y)$ is the PSF of the imaging sensor, $P S F_{I P}(x, y)$ is equal to the image processing part and $P S F_{\text {compr }}(x, y)$ covers the influence of using of an image compression method. Each of these steps can distort the acquired image and can change it irreversibly. When a lossless compression algorithm is used the appropriate point spread function is equal to the Dirac impulse.

The application of lossy compression methods deforms the point spread function of the imaging system, of course [5]. It is therefore necessary to consider the compression intensity that is used. The deformation of the point spread function is used as the most important quality criterion in this paper. In most cases, the imaging system can be considered as a lin- ear and space invariant case. Other systems can be described as piecewise linear and space invariant [6].

\section{Image data and point spread function model}

Astronomical images are a special class of images. They have different parameters from multimedia image classes. The most important distinctions are:

- high bit depth (up to 16 bits)

- grayscale and color different from the multimedia RGB system

- significant noise level

- sophisticated algorithms for processing astronomical images

Three images have been chosen as typical representatives of astronomical images. The first is an image acquired by a wide field camera of the BOOTES experiment (Burst Observer and Optical Transient Exploring System) [1]. The image captures the neighborhood of the M7 with objects (stars) with the full width at half maximum (FWHM) of approximately a few pixels (see Figure 1). The other two images can be classified in the DEEP SKY class. These images are acquired with longer focus optics. Image M42 is with a satellite tray and image M51 is in the red filter (see Figure 1). These images are stored in the DEIMOS image database [2].

A 2D Gaussian or Moffat model of a Point Spread Function (PSF) can be used for space invariant linear systems without dominant influence of image aberrations [8]. 
a)

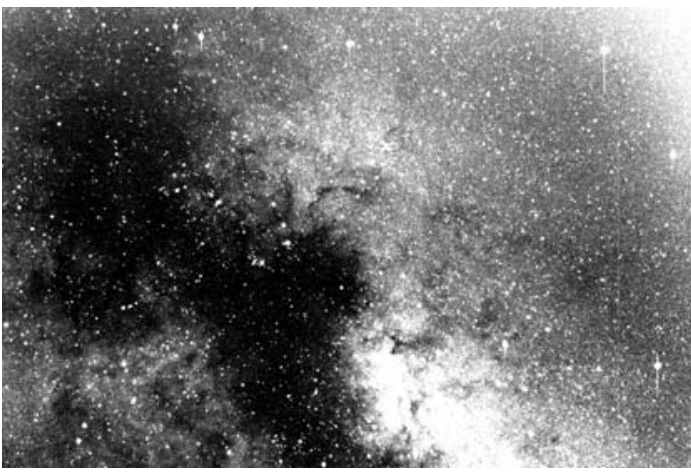

b)

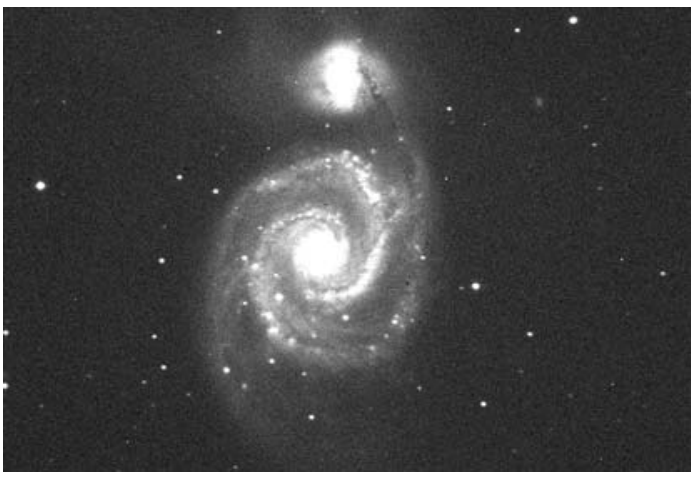

c)

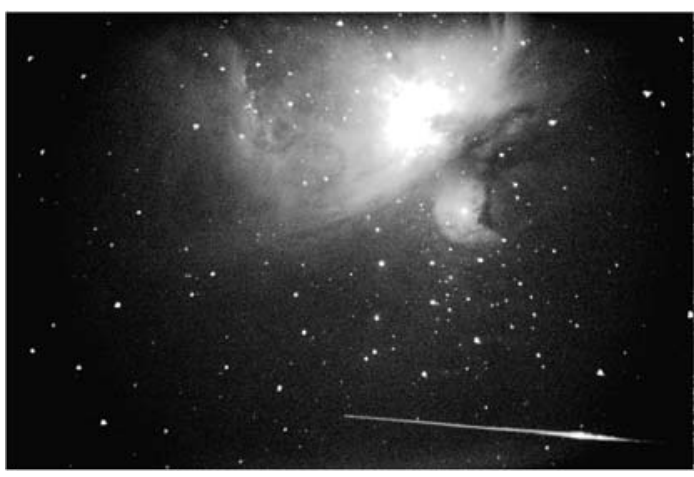

Fig. 1: Input image data from the DEIMOS database: a) Image from wide field camera M7 and the Milky Way with many objects (size smaller than 10 pixels)

b) M51 galaxy in red filter. DEEP SKY image with bigger objects

c) M42 nebulae with a satellite tray. Image obtained from the DEEP SKY camera of the BOOTES system

\section{Results and measurements}

The JAVA implementation of the Astronomical Context Coder [7] has been used for the influence of lossy compression on PSF deformation. This software package contains the JPEG2000 standard with an extension for 16bit images. The criteria were chosen for compressed image quality evaluation. These criteria are based on a description of the image function with respect to the deformation of objects in the image and also the precision of the photometric and astrometric algorithms. The following set of criteria has been chosen:
- Point spread function deformation measured by the Moffat function fit ( $\beta$ parameter, see Figure $2 \mathrm{a}$ ).

- Object position error expressed as the object center of mass (in pixels, see Figure $2 \mathrm{~b}$ ).

- Object flux error. This flux is defined as the sum of the image function over the object expressed as sensor irradiation (the background value is removed) (as a percentage, see Figure 2c).

a)

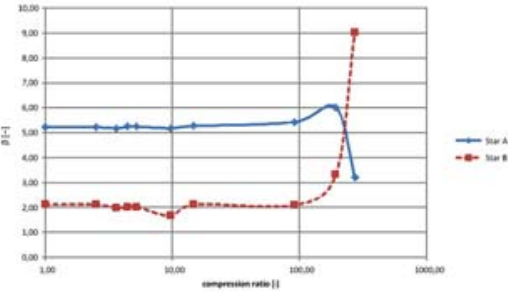

b)

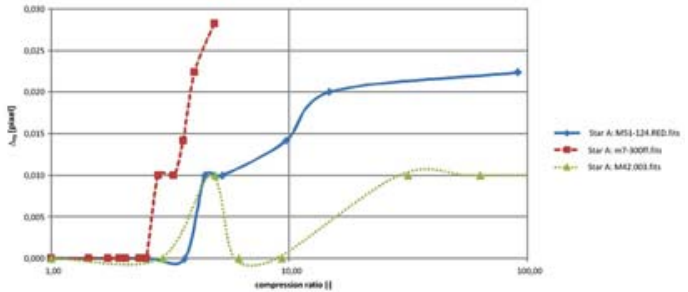

c)

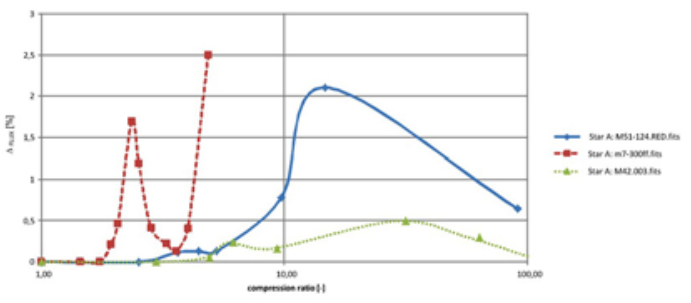

Fig. 2: Influence of lossy compression on Moffat parameter deformation a), object position error b) and change of the object with the compression ratio $\mathrm{c}$ )

The IRAF software package has been chosen for image analyzing [3]. Two objects with different brightness have been selected to demonstrate the results. These objects have different FWHM and they are therefore not equally sensitive to damage by lossy compression methods.

\section{Conclusion}

Compression and processing of astronomical images are different tasks from the classical way known from multimedia technology. It is not possible to use a setup optimized for human vision. The influence of the lossy compression standard JPEG 2000 on the distortion of the object profile has been verified in this paper. The profile is closely related to the point spread function of the imaging system. It can be said that the influence of lossy compression is more significant for faint objects with FWHM (equal object area) 
not exceeding a few pixels (image M7-300ff.fits). Special quality criteria and acceptable distortion are necessary for defining the application of a lossy compression standard.

\section{Acknowledgement}

This work has been supported by grant No. P102/10/1320 "Research and modeling of advanced methods of image quality evaluation" of the Grant Agency of the Czech Republic and by research project MSM 6840770014 "Research of perspective information and communication technologies" of MSMT of the Czech Republic.

\section{References}

[1] BOOTES, Burst Observer and Optical Transient Exploring System. 2011. http://www.laeff.esa.es/BOOTES/ing/index.html

[2] Fliegel, K., Klíma, M., Páta, P.: New open source image database for testing and optimization of image processing algorithms, In Optics, Photonics, and Digital Technologies for Multimedia Applications. SPIE Proceedings, Vol. 7 723, 2010.

[3] IRAF, Image Reduction and Analysis Facility. 2011. [online] http://www.iraf.noao.edu
[4] ISO/IEC 15444-1:2000: JPEG2000 Image Coding System (core coding system). 2000. [online] http://www.jpeg.org/FCD15444-1.htm

[5] Páta, P., Hanzlík, P., Schindler, J., Vítek, S.: Influence of Lossy Compression Techniques on Processing Precision of Astronomical Images, $6^{\text {th }}$ IEEE ISSPIT conference, Athens, Greece : 2005.

[6] Řeřábek, M., Páta, P.: Astronomical Image Compression Techniques Based on ACC and KLT Coder, Proceedings of the 7th Integral/BART Workshop, IBWS2010, Acta Polytechnica, 2011.

[7] Schindler, J., Páta, P., Klíma, M., Páta, P.: Advanced Processing of Images Obtained from Wide-field Astronomical Optical Systems, Proceedings of the 7th Integral/BART Workshop, IBWS2010, Acta Polytechnica, 2011.

[8] Starck, J. L., Murtagh, F.: Astronomical Image and Data Analysis, Springer, 2002.

Petr Páta

Department of Radioengineering Faculty of Electrical Engineering Czech Technical University in Prague Technická 2, Prague, Czech Republic 